\title{
Companion canines hold clues for cancer research
}

\author{
Research using pet dogs as animal models of cancer is helping to inform treatments for human patients - and \\ vice versa.
}

\section{Alla Katsnelson}

1 $s$ a veterinarian in private practice in the early 1990s, Cheryl London found it especially distressing to tell pet owners that their dogs had cancer. There were almost no treatments available. So she went back to school to study veterinary oncology, a specialty that was still relatively new.

Soon afterwards, while doing her doctorate in immunology at Harvard University, London made a surprising discovery: dog mast cell tumors shared a molecular pathology with a type of human gastrointestinal cancer. Like the human cancer, these dog tumors are regulated by the oncogene c-kit, and a significant percentage of them carry mutations in the gene, she found.

It was the first driver mutation identified in spontaneous canine cancer, and human cancer researchers hadn't much considered the idea that there might be a direct alignment between tumors in humans and another species. Meanwhile, pharmaceutical companies were beginning to home in on specific genetic alterations with targeted cancer therapies. A drug company called Sugen (later acquired by Pfizer) was testing whether drugs called tyrosine kinase inhibitors could treat gastrointestinal cancer by targeting c-kit mutations. When London started her own lab at the University of California, Davis, she reached out to the company to see if she could test some of the inhibitors in dogs.

In 2000 , they sent her toceranib, a backup compound to one they hadn't trialed in humans yet called sunitinib. Over the next few years, her lab's clinical tests with the drug in dogs showed that toceranib could delay or prevent the progression of these common tumors. Her results helped inform human clinical trials of sunitinib, which the US Food and Drug Administration (FDA) approved in 2006. Three years later, toceranib (Palladia) became the first cancer therapy to be approved by the agency specifically in dogs.

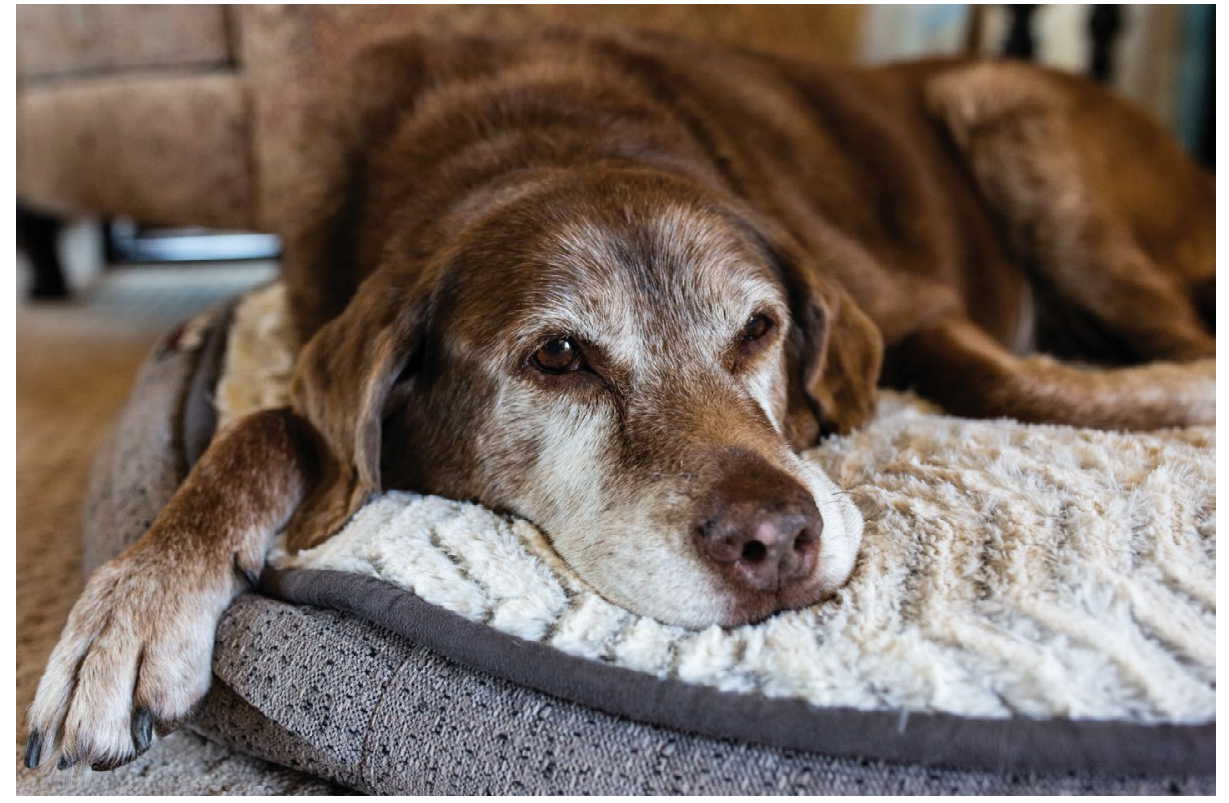

A natural model | Our companions share many of the same age and environmental risk factors for cancer as we do, affording researchers and veterinarians a unique model in which to study spontaneously developing tumors. Credit: Cavan Images / Alamy Stock Photo

Toceranib is a poster child for the value that investigating spontaneous cancers in pet dogs can bring to human cancer research, and vice versa. Although the link between companion canine and human cancer had been probed before, interest in this comparative approach has grown dramatically in the decade since toceranib's approval. With support from the National Cancer Institute (NCI), pharmaceutical companies, and philanthropic organizations aligned with both humans and pets, the infrastructure is finally emerging for cancer researchers working across species to make major contributions to therapeutics development, says David Vail, a professor of comparative oncology at the University of Wisconsin-Madison. That work is providing important insights into human drug development, but it is also driving the development of new treatments for dogs. "At the end of the day, I'm a veterinarian and I want to help my patients, says Vail. "We try to ensure there's a bidirectional flow of discovery."

And the investment is paying off - for both species. Researchers have identified at least seven types of cancer occurring in pet dogs that share molecular features with human cancers. There have been perhaps a dozen human clinical trials for cancer therapies that have directly benefited from clinical data collected from companion canines. Most treatments for canine cancers are still human drugs used off-label by veterinary oncologists, but the arsenal of dog-specific medicines is increasing. Recently two therapies for human cancer research programs received conditional FDA approval to treat lymphoma in 
dogs - rabacfosadine (Tanovea-CA) in 2017 and verdinexor (Laverdia-CA) early this year - and several more are advancing through clinical trials.

"This has been simmering for a very long time - until the last 10 years or so, when it has really exploded," says London, who is now a professor of comparative oncology at Tufts University. "It's really all gelling together now."

\section{The canine advantage}

Companion dogs have a slew of advantages as a model for cancer. Perhaps the most significant is that, like humans, dogs develop spontaneous cancers that often emerge with age. "A lot of it has to do with the fact that these are naturally arising tumors in a heterogeneous population," says Vail. In mouse models, meanwhile, tumors are generally induced or transplanted, which means that their development almost certainly follows a different trajectory and occurs in a different microenvironment than those that develop spontaneously. And because most mice used in cancer research are inbred, they have little genetic variability; no matter how many you use in a study, to some extent they correspond to a single person.

Dogs that develop cancer also have intact immune systems, just as humans generally do - for an artificial tumor to grow in a rodent cancer model, the animals must often have artificial or depleted immune systems. On the molecular level, several types of dog and human tumors have similar features under the microscope. And, basic features of the human and canine immune systems - for example, markers on different immune cell types - tend to be closely aligned, explains Amy LeBlanc, director of the comparative oncology program at the NCI. Because cancer is in part a failure of immune surveillance, these similarities are important, and particularly crucial for investigating immunotherapies.

On a more basic level, a dog's lifespan of 8-14 years is more comparable to that of human than the 1-3 years that mice live, yet short enough to realistically assess a drug's or a vaccine's long-term effects. Body size is a key feature, too - researchers don't need to reinvent clinical tools such as imaging machines or surgical devices used with humans to study dogs, and the scales at which the treatments work are also roughly parallel. And as human companions, dogs are subject to many of the same environmental factors that may contribute to cancer development. "They're breathing the same air, they're walking on lawns treated with the same chemicals, they're drinking the same water," says Douglas Thamm, a professor of veterinary oncology at Colorado State University.

Finally, because there isn't a clear standard of care for treating most canine cancers, companion dogs often enter a clinical trial before they have undergone other treatments, which makes it easier to evaluate a drug's response and side effects. In contrast, by the time a person receives an experimental therapy, their cancer is generally advanced and their immune system depleted. Researchers can also biopsy canine tumors multiple times as a treatment progresses, allowing them to monitor its effects. That's impossible in mice - tissue sampling generally means sacrificing the animals - and is not always possible in humans, either. "There's really no replacement for repeated serial biopsies in naturally occurring tumor tissue to really make you confident that the drug is doing what you think it's doing," says LeBlanc.

\section{Lessons learned}

To some extent, says LeBlanc, this cross-species approach is nothing new. "Since time immemorial, veterinarians have been trying to learn from our human counterparts," she says. But it took a few decades for the mindset to catch on as a well-defined strategy. Veterinarians first started characterizing canine tumors back in the 1960s and 70s, observing significant similarities between dogs and humans, says Thamm. But he and others credit three pioneering veterinary oncologists in the 1980s and 1990s - Edward Gillette and Stephen Withrow at Colorado State University and Greg MacEwen at the University of Wisconsin - with realizing that studies in dogs could serve as a bridge between animal models and human translation. Gillette pioneered the use of radiation oncology in dogs while Withrow developed a surgical technique for removing tumors causing osteosarcoma, an aggressive bone cancer common in dogs, that preserved their limbs; pediatric oncologists soon adopted this limb-sparing procedure in children with osteosarcoma. MacEwen, meanwhile, investigated an injectable immune-stimulating molecule called mifamurtide (Mepact) to treat metastatic canine osteosarcoma. That trial's positive results paved the way for studies on that drug's efficacy against osteosarcoma in children, eventually leading to its approval in the European Union in 2009.

Such studies drew interest from the NCI, which launched the Comparative Oncology program in 2003 to systematically explore cross-pollination between canine and human cancer research. "I think once people saw there was actually buy-in from

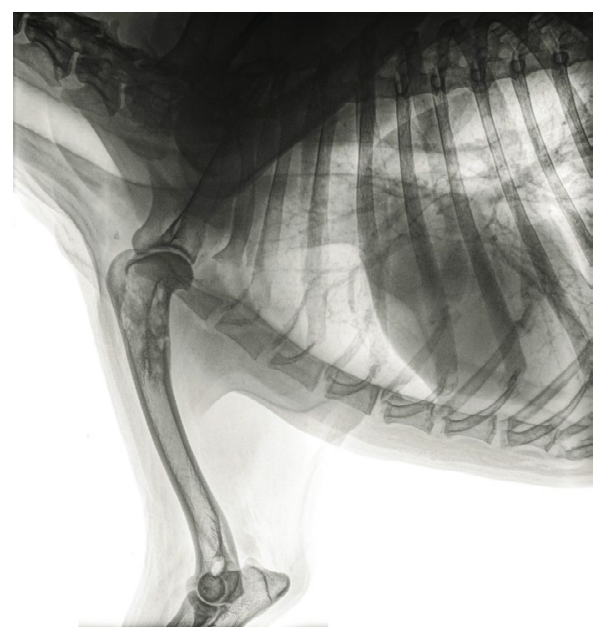

In the bones | Osteosarcoma, seen here in the upper humerus, can afflict both canines and people and was one of the first cancers explored in pet dogs to yield translational insights for human patients. Credit: Henk Vrieselaar / Alamy Stock Photo

the National Cancer Institute, that lent some legitimacy to the idea that this is something people should look at," says Thamm. Today, most major US veterinary schools have oncology programs, and there is increasing cross-pollination between veterinary and human clinical trial networks. That's not to say though that the aims of the approach are universally recognized among human cancer researchers. "It never surprises me: There's always somebody who puts up their hand after a talk and asks, 'Can you go back and explain how you induced cancer in the patients?"” says Vail. "And we go, "No, these are spontaneous, naturally occurring cancers."

Osteosarcoma was the starting point for probing the canine-human cancer connection. Usually affecting the long bones of limbs, the disease sickens about 10,000 dogs and 800-900 children and young adults in the US each year. Its frequency in dogs places a heavy burden on pets and their owners, and its rarity in humans raises challenges for conducting clinical trials. Other cancer types also have canine - human correlates: hemangioma in dogs is similar to angiosarcoma in humans, for example, and oral melanoma in dogs resembles mucosal melanoma in humans. But as veterinary researchers begin defining the molecular and genetic features of canine cancers, they are also identifying dog and human cancers that may affect a different tissue type but share molecular features. In essence, cross-species comparisons 
allow researchers to home in on the shared mechanisms, explains Geoffrey Wood, an assistant professor of veterinary pathology at the University of Guelph. "These different species act as sort of biological filters, to pick out the most biologically relevant mutations," he says.

The c-kit mutation targeted by toceranib and sunitinib offers an early example, but several others have emerged. For example, $85 \%$ of canine bladder cancers sport a specific mutation in the BRAF gene, a mutation also carried in a subset of human cancers across tissue types, including melanoma, thyroid cancer, and colon cancer. That link provides an opportunity to develop therapies for both species that target the mutation, but it also offers a pathway for dissecting the underlying biological understanding of how specific genes and signaling pathways drive cancer, says London. "We're getting more and more to the point where the histopathologic description of the disease is less important, and instead we are trying to match the diseases on a genetic level."

To better exploit these shared alterations, the field is knee-deep in an effort to characterize the genetic and molecular features of canine cancers. "We are working feverishly to get a better handle on that molecular landscape," LeBlanc says. Her team, for example, is conducting extensive molecular profiling on canine osteosarcoma tumors in order to match up subgroups in both canine and pediatric patients with biologically similar cancers - and eventually, to match drugs to those subgroups. London's group recently identified a mutation in the gene for dystrophin that's present in about half of canine osteosarcoma tumors and also pops up in several human cancers. "It seems to be correlated with an aggressive biology, but we don't have any idea what it's doing yet," she says.

Other work takes an even broader view. Researchers at the Sanger Institute are launching a study sequencing about 100 known cancer genes in multiple canine tumors in order to look for similarities in molecular profiles that can then be compared with human cancers.
Building out the genomics knowledge base should in turn accelerate the development of therapies that target specific cancer mutations in dogs, says Louise van Der Weyden, a senior staff scientist in experimental cancer genetics at the Sanger Institute. Last year, NCI launched a platform called the Integrated Canine Data Commons, that standardizes data to allow researchers to analyze molecular features across the growing collection of data sets. The bottleneck right now, says London, is synthesizing it: "Once you have the genomic data on a tumor, how do you use it appropriately to design a therapeutic regimen?"

The comparative approach isn't limited to dogs. A few efforts are also looking at cats. The comparison is not quite as straightforward, says Vail, because cats have very different metabolisms compared to humans and dogs and they also tend to be less willing to undergo regular procedures. But one project is investigating head and neck squamous cancer, which is similar in cats and people. Another is looking at mammary cancer, which is much more malignant in cats than in dogs. "So it kind of models like the worst of the worst in humans," says Wood. Bringing cats into the comparative fold means developing some of the same tools that have emerged for studying dogs. Van der Weyden has even extended comparisons to horses. "If it's something that spontaneously happens in a decent proportion of the species, that to me is a readymade model and we should look at it," she says.

\section{A fetching future}

One area of comparative oncology gaining momentum is immunotherapy. Researchers are still figuring out how best to wield treatments that target the human immune system; because dogs have a broadly similar immune system, "they may be uniquely useful for this particular line of inquiry," says Thamm. Molecules called checkpoint inhibitors, which release the brakes on $\mathrm{T}$ cell immune response against tumors, have proven especially promising in human cancer treatment, and veterinary researchers are trying to develop canine versions of them. Vail's team, meanwhile, is testing combinations of immunotherapies, and London's lab is looking for combinations of chemotherapy and immunotherapy that allows veterinarians to use less of the former, to minimize toxicity in the animals.

Multiple vaccines are also in development. For example, Jeffrey Bryan at the University of Missouri and his colleagues, working with ELIAS Animal Health, which develops canine cancer immunotherapies, recently showed in a small study that a vaccine made from $\mathrm{T}$ cells raised against a dog's own tumor, paired with injections of an immune stimulating molecule called interleukin-2, appears to keep osteosarcoma in check. "Of the 10 dogs that received every part of the protocol, five of them were alive two years later," says Bryan. "Honestly, for osteosarcoma, we just don't have very many two-year survivors." Other approaches for targeting the immune system are also in the works. Thamm is working with a company called Corvus Pharmaceuticals to test a small molecule that targets T cell lymphoma. His team's results showing the molecule's anti-tumor activity in dogs is accelerating the trajectory of human clinical trials, he says.

In a very different type of project, Thamm is currently leading the canine component of an ambitious trial testing a preventative vaccine that recognizes antigens from multiple types of cancer. The idea of a vaccine that can prevent a wide range of cancers in people has been controversial, and demonstrating its efficacy in humans - especially considering our extended lifespans - is a daunting proposition. But if he and his colleagues can demonstrate its efficacy in dog breeds with a high risk of cancer, he says, "boy, this would really provide evidence that something like this should be looked at in people."

\section{Alla Katsnelson $\bowtie$ \\ Freelance science writer, Massachusetts, USA.

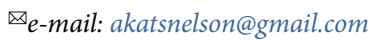

Published online: 17 March 2021

https://doi.org/10.1038/s41684-021-00741-y 